\title{
DRINFELD FUNCTOR AND FINITE-DIMENSIONAL REPRESENTATIONS OF YANGIAN
}

\author{
TOMOYUKI ARAKAWA
}

\begin{abstract}
We extend the results of Drinfeld on the Drinfeld functor to the case $\ell \geq n$. We present the character of finite-dimensional representations of the Yangian $Y\left(\mathfrak{s l}_{n}\right)$ in terms of the Kazhdan-Lusztig polynomials as a consequence.
\end{abstract}

\section{INTRODUCTION}

In this article we study the representations of the Yangian $Y\left(\mathfrak{s l}_{n}\right)$. The Yangian is a quantum group introduced by V. G. Drinfeld (D1). The parameterization of the simple finite-dimensional representations of $Y\left(\mathfrak{s l}_{n}\right)$ was obtained in [D3] by the sequences of monic polynomials $Q(u)=\left(Q_{1}(u), \ldots, Q_{n-1}(u)\right)$ called the Drinfeld polynomials. Furthermore, he has constructed in D2 a functor from the category $\mathcal{C}_{\mathcal{H}_{\ell}}$ of finite-dimensional representations of the degenerate affine Hecke algebra $\mathcal{H}_{\ell}$ to the category $\mathcal{C}_{Y\left(\mathfrak{s l}_{n}\right)}$ of finite-dimensional representations of $Y\left(\mathfrak{s l}_{n}\right)$. This functor is called the Drinfeld functor. It was stated in [D2] that as well as the classical Frobenius-Schur duality, the Drinfeld functor gives the categorical equivalence between $\mathcal{C}_{\mathcal{H}_{\ell}}$ and the certain subcategory of $\mathcal{C}_{Y\left(\mathfrak{s l}_{n}\right)}$ in the case $\ell<n$. Chari-Pressley generalized this duality to the case between the affine Hecke algebra and the quantum affine algebra. They proved that the categorical equivalence holds in this case as well provided that $\ell<n(\mathrm{CP} 2]$.

However, due to the restriction $\ell<n$, the above categorical equivalence does not describe all the finite-dimensional representations of the Yangian $Y\left(\mathfrak{s l}_{n}\right)$. In particular, even the characters of finite-dimensional representations of $Y\left(\mathfrak{s l}_{n}\right)$ have not been known, except for the case $n=2(\overline{\mathrm{CP}} 3)$ and the special class of the representations called tame ( NT1]).

The main purpose of this article is to extend the Drinfeld's results [D2] to the case $\ell \geq n$. To be more precise, we first show the followings without restriction $\ell<n$ :

1. The Drinfeld functor sends the standard modules of $\mathcal{H}_{\ell}$ to zero or the highest modules of $Y\left(\mathfrak{s l}_{n}\right)$ (Theorem 8).

2. The Drinfeld functor sends the simple modules of $\mathcal{H}_{\ell}$ to zero or the simple modules of $Y\left(\mathfrak{s l}_{n}\right)$ (Theorem 10).

Here the standard modules are certain induced $\mathcal{H}_{\ell}$-modules which have unique simple quotients (see subsection 1.4). We also determine the explicit images of the standard modules. It turns out that the highest weight modules obtained as the images of the standard modules are those tensor product modules of the evaluation representations studied in AK]. We note that any simple $Y\left(\mathfrak{s l}_{n}\right)$-module is isomorphic to the image of a simple $\mathcal{H}_{\ell}$-module for some $\ell$. 
Further, combining the above results with that of the representation theory of $\mathcal{H}_{\ell}$, we state the following:

3. The multiplicity formula of $Y\left(\mathfrak{s l}_{n}\right)$ expressed in terms of the Kazhdan-Lusztig polynomials (Theorem 15)日.

This is the result of considering the composition $D_{\ell} \circ F_{\lambda}$ of the two exact functors, where $F_{\lambda}$ is the functor from the Bernstein-Gelfand-Gelfand category $\mathcal{O}_{r}$ of the complex Lie algebra $\mathfrak{g l}_{r}$ to the category $\mathcal{C}_{\mathcal{H}}$, obtained by Suzuki and the author in AS.

\section{Preliminaries}

1.1. Yangian. Let $n$ be a positive integer. First we review some fundamental facts about the algebra structure of the Yangian $Y\left(\mathfrak{s l}_{n}\right)$. Our main references are D1, D3, MNO] and we basically follow the notation of [MNO].

Let

$$
R(u)=1-\frac{P}{u} \in \operatorname{End}\left(\mathbb{C}^{n} \otimes \mathbb{C}^{n}\right),
$$

where $P$ is the permutation operator in $\mathbb{C}^{n} \otimes \mathbb{C}^{n}$ and $u$ is a parameter. Let $E_{i j} \in$ $\mathfrak{g l}_{n}=\mathfrak{g l}_{n}(\mathbb{C})$ denote the usual matrix operator on $\mathbb{C}^{n}$. The Yangian $Y\left(\mathfrak{g l}_{n}\right)$ is the unital associative algebra over $\mathbb{C}$ with generators $t_{i j}^{(k)}(1 \leq i, j \leq n, k=0,1,2, \ldots)$ and the defining relations

$$
R_{12}(u-v) t_{1}(u) t_{2}(v)=t_{2}(v) t_{1}(u) R_{12}(u-v),
$$

where

$$
\begin{gathered}
t(u)=\sum_{i, j} t_{i j}(u) \otimes E_{i j} \in Y\left(\mathfrak{g l}_{n}\right) \otimes \operatorname{End}\left(\mathbb{C}^{n}\right), \\
t_{i j}(u)=\sum_{d=0}^{\infty} t_{i j}^{(d-1)} u^{-d} \in Y\left(\mathfrak{g l}_{n}\right)\left[\left[u^{-1}\right]\right]
\end{gathered}
$$

Here we put $t_{i j}^{(-1)}=\delta_{i j}$ id and both sides of (1.1.1) are regarded as elements of $Y\left(\mathfrak{g l}_{n}\right)\left(\left(v^{-1}\right)\right)\left[\left[u^{-1}\right]\right] \otimes \operatorname{End}\left(\mathbb{C}^{n}\right) \otimes \operatorname{End}\left(\mathbb{C}^{n}\right)$ and the subindexes of $t(u)$ and $R(u)$ indicate to which copy of $\operatorname{End}\left(\mathbb{C}^{n}\right)$ these matrices correspond.

The defining relations (1.1.1) are equivalent to the following relations:

$$
\left[t_{i j}^{(r)}, t_{k l}^{(s-1)}\right]-\left[t_{i j}^{(r-1)}, t_{k l}^{(s)}\right]=t_{k j}^{(r-1)} t_{i l}^{(s-1)}-t_{k j}^{(s-1)} t_{i l}^{(r-1)}
$$

$\left(1 \leq i, j, k, l \leq n, r, s \in \mathbb{Z}_{\geq 0}\right)(\mathrm{MNO})$.

The algebra $Y\left(\mathfrak{g l}_{n}\right)$ is a Hopf algebra with coproduct

$$
\Delta: t_{i j}(u) \mapsto \sum_{a=1}^{n} t_{i a}(u) \otimes t_{a j}(u),
$$

antipode $S(t(u))=t(u)^{-1}$ and counit $\varepsilon(t(u))=1$.

Let $U\left(\mathfrak{g l}_{n}\right)$ denote the universal enveloping algebra of the Lie algebra $\mathfrak{g l}_{n}$. The algebra $U\left(\mathfrak{g l}_{n}\right)$ is considered as a subalgebra of $Y\left(\mathfrak{g l}_{n}\right)$ by the inclusion homomorphism defined by

$$
\begin{aligned}
U\left(\mathfrak{g l}_{n}\right) & \longrightarrow Y\left(\mathfrak{g l}_{n}\right) \\
E_{i j} & \longmapsto t_{i j}^{(0)} .
\end{aligned}
$$

\footnotetext{
1 After completing this article, the author was notified that E. Vasserot obtained the similar formula in terms of intersection cohomologies in the case of the quantum affine algebra by geometrical method ([preprint, math.QA/9803024]).
} 
On the other hand, for $a \in \mathbb{C}$, the map

$$
\begin{aligned}
\mathrm{ev}_{a}: Y\left(\mathfrak{g l}_{n}\right) & \longrightarrow U\left(\mathfrak{g l}_{n}\right) \\
t_{i j}(u) & \longmapsto \delta_{i j}+\frac{E_{i j}}{u-a}
\end{aligned}
$$

defines an algebra homomorphism. For a $\mathfrak{g l}_{n}$-module $V$, let $\mathrm{ev}_{a}(V)$ denote the $Y\left(\mathfrak{g l}_{n}\right)$-module obtained by pulling $V$ back by (1.1.4).

The quantum determinant $q \operatorname{det} t(u)$ is defined as

$$
\operatorname{qdet} t(u)=\sum_{w \in W_{n}}(-1)^{\operatorname{sgn}(w)} t_{w(1), 1}(u) t_{w(2), 2}(u-1) \cdots t_{w(n), n}(u-n+1),
$$

where $W_{n}$ denotes the symmetric group $\mathfrak{S}_{n}$. The coefficients of the quantum determinant are algebraically independent and generate the center $Z\left(Y\left(\mathfrak{g l}_{n}\right)\right)$ of $Y\left(\mathfrak{g l}_{n}\right)$.

For a formal series $f(u)=1+f_{1} u^{-1}+f_{2} u^{-2}+\cdots \in \mathbb{C}\left[\left[u^{-1}\right]\right]$, the multiplication

$$
t(u) \longmapsto f(u) t(u)
$$

defines an automorphism of $Y\left(\mathfrak{g l}_{n}\right)$. It is known that the Yangian $Y\left(\mathfrak{s l}_{n}\right)$ can be defined as the subalgebra of $Y\left(\mathfrak{g l}_{n}\right)$ consisting of elements fixed by all automorphisms of the form (1.1.5) (MNO). One has a tensor product decomposition

$$
Y\left(\mathfrak{g l}_{n}\right) \cong Z\left(Y\left(\mathfrak{g l}_{n}\right)\right) \otimes Y\left(\mathfrak{s l}_{n}\right)
$$

Hence any $Y\left(\mathfrak{g l}_{n}\right)$-module can be considered as a $Y\left(\mathfrak{s l}_{n}\right)$-module.

Let

$$
F_{i} Y\left(\mathfrak{g l}_{n}\right):=\sum_{\substack{k \in \mathbb{Z}_{\geq 0} \\ 1 \leq i_{a}, j_{a} \leq n}} \sum_{\substack{r_{i} \in \mathbb{Z}_{\geq 0} \\ r_{1}+\cdots+r_{k} \leq i}} \mathbb{C} t_{i_{1} j_{1}}^{\left(r_{1}\right)} \ldots t_{i_{k} j_{k}}^{\left(r_{k}\right)} \quad\left(i \in \mathbb{Z}_{\geq 0}\right) .
$$

Then, by 1.1.2), $F_{i} Y\left(\mathfrak{g l}_{n}\right) \cdot F_{j} Y\left(\mathfrak{g l}_{n}\right) \subset F_{i+j} Y\left(\mathfrak{g l}_{n}\right)$, and (1.1.7) defines a filteration on $Y\left(\mathfrak{g l}_{n}\right)$. Let gr $Y\left(\mathfrak{g l}_{n}\right)$ denote the corresponding the graded algebra;

$$
\operatorname{gr} Y\left(\mathfrak{g l}_{n}\right)=\bigoplus_{i \in \mathbb{Z}_{\geq 0}} F_{i} Y\left(\mathfrak{g l}_{n}\right) / F_{i-1} Y\left(\mathfrak{g l}_{n}\right) \quad\left(F_{-1} Y\left(\mathfrak{g l}_{n}\right)=0\right) .
$$

Then, gr $Y\left(\mathfrak{g l}_{n}\right)$ is isomorphic to $U\left(\mathfrak{g l}_{n}[t]\right)$, where $U\left(\mathfrak{g l}_{n}[t]\right)$ is the universal enveloping algebra of the polynomial current algebra $\mathfrak{g l}_{n}[t]:=\mathfrak{g l}_{n} \otimes \mathbb{C}[t]$ with $\mathbb{Z}_{\geq 0}$-grading such that the degree of the element $X \otimes t^{r}\left(X \in \mathfrak{g l}_{n}\right)$ equals $r$ (MNO).

1.2. Drinfeld polynomials. In this subsection we review the classification theory of finite-dimensional simple $Y\left(\mathfrak{s l}_{n}\right)$-modules studied by Drinfeld ( [D3], see also [CP1, Mol])

A representation $V$ of $Y\left(\mathfrak{g l}_{n}\right)$ is called highest weight if there exists a cyclic vector $v$ such that $t_{i j}(u) \cdot v=0(1 \leq i<j \leq n)$ and $t_{i i}(u-i) \cdot v=\zeta_{i}(u) v$ $(1 \leq i \leq n)$ for some formal series $\zeta_{i}(u) \in 1+u^{-1} \mathbb{C}\left[\left[u^{-1}\right]\right]$. The vector $v$ is called the highest weight vector of $V$ and the sequence $\zeta(u)=\left(\zeta_{1}(u), \ldots, \zeta_{n}(u)\right)$ is called the highest weight of $V$. The central element qdet $t(u)$ acts as a constant $\zeta_{1}(u) \zeta_{2}(u-1) \ldots, \zeta_{n}(u-n+1)$ on a highest weight module $V$. As in the classical Lie algebra theory, any highest weight $Y\left(\mathfrak{g l}_{n}\right)$-module has a unique simple quotient, in which the image of its highest weight vector is nonzero.

It is known by Drinfeld that a simple highest weight module of $Y\left(\mathfrak{g l}_{n}\right)$ is finitedimensional if and only if there exists a sequence of monic polynomials $Q(u)=$ 
$\left(Q_{1}(u), \ldots, Q_{n-1}(u)\right)$ such that

$$
\frac{\zeta_{k}(u)}{\zeta_{k+1}(u+1)}=\frac{Q_{k}(u+1)}{Q_{k}(u)}
$$

for $k=1, \ldots, n-1$. A theorem of Drinfeld states that there is a one-to-one correspondence between the finite-dimensional simple $Y\left(\mathfrak{s l}_{n}\right)$-modules and the sequences of monic polynomials $Q(u)=\left(Q_{1}(u), \ldots, Q_{n-1}(u)\right)$ defined by (1.2.1) (ID3). The $Q(u)$ are called Drinfeld polynomials.

Remark 1. The standard symbol for the Drinfeld polynomials is $P(u)$. However, this symbol for a polynomial is reserved for the Kazhdan-Lusztig polynomials in this article.

1.3. Degenerate Affine Hecke Algebra. Let $\ell$ be a positive integer. Let $\mathfrak{h}_{\ell}$ be the Cartan subalgebra of $\mathfrak{g l}_{\ell}$, which consists of the diagonal matrices. Define a basis $\left\{\epsilon_{i}\right\}_{i=1}^{\ell}$ of $\mathfrak{h}_{\ell}$ by putting $\epsilon_{i}=E_{i i}$. The dual space $\mathfrak{h}_{\ell}^{*}$ is identified with $\mathfrak{h}_{\ell}$ via the inner product $\left\langle\epsilon_{i}, \epsilon_{j}\right\rangle=\delta_{i j}$.

Let $R_{\ell}$ be the root system of $\mathfrak{g l}_{\ell}$;

$$
\begin{aligned}
& R_{\ell}=\left\{\alpha_{i j}=\epsilon_{i}-\epsilon_{j} \mid 1 \leq i \neq j \leq \ell\right\}, \\
& R_{\ell}^{+}=\left\{\alpha_{i j} \in R_{\ell} \mid i<j\right\}, \\
& \Pi_{\ell}=\left\{\alpha_{i}=\alpha_{i i+1} \mid i=1, \ldots, \ell-1\right\},
\end{aligned}
$$

where $R_{\ell}^{+}$is the set of positive roots and $\Pi_{\ell}$ is the set of simple roots. Let $\rho=$ $\frac{1}{2} \sum_{\alpha \in R_{\ell}^{+}} \alpha$. We identify the coroots with the roots throughout this article.

Let $s_{\alpha} \in W_{\ell}$ denote the reflection corresponding to $\alpha \in R_{\ell}$;

$$
s_{\alpha} \cdot \lambda=\lambda-\lambda(\alpha) \alpha \quad\left(\lambda \in \mathfrak{h}_{\ell}^{*}\right) .
$$

Put $s_{i j}=s_{\alpha_{i j}}\left(\alpha_{i j} \in R_{\ell}\right)$ and $s_{i}=s_{\alpha_{i}}\left(\alpha_{i} \in \Pi_{\ell}\right)$.

Let $S\left(\mathfrak{h}_{\ell}\right)$ be the symmetric algebra of $\mathfrak{h}_{\ell}$, which is isomorphic to the polynomial ring over $\mathfrak{h}_{\ell}^{*}$.

The degenerate affine Hecke algebra $\mathcal{H}_{\ell}$ of $G L_{\ell}($ [D2]) is the associative algebra over $\mathbb{C}$ such that

$$
\mathcal{H}_{\ell} \cong \mathbb{C}\left[W_{\ell}\right] \otimes S\left(\mathfrak{h}_{\ell}\right)
$$

as a vector space, the subspaces $\mathbb{C}\left[W_{\ell}\right] \otimes \mathbb{C}$ and $\mathbb{C} \otimes S\left(\mathfrak{h}_{\ell}\right)$ are subalgebras of $\mathcal{H}_{\ell}$, and the following relations hold in it;

$$
s_{\alpha} \cdot \xi-s_{\alpha}(\xi) \cdot s_{\alpha}=-\alpha(\xi)\left(\alpha \in \Pi_{\ell}, \xi \in \mathfrak{h}_{\ell}\right),
$$

where the elements $\xi \in \mathfrak{h}_{\ell}$ and $w \in W_{\ell}$ are identified with $1 \otimes \xi \in \mathcal{H}_{\ell}$ and $w \otimes 1 \in \mathcal{H}_{\ell}$ respectively. One has

$$
\mathcal{H}_{\ell}=\mathbb{C}\left[W_{\ell}\right] \cdot S\left(\mathfrak{h}_{\ell}\right)=S\left(\mathfrak{h}_{\ell}\right) \cdot \mathbb{C}\left[W_{\ell}\right] .
$$

We put $\mathcal{H}_{0}=\mathbb{C}$ for convenience.

The center $Z\left(\mathcal{H}_{\ell}\right)$ of this algebra equals the $W_{\ell}$-invariant polynomials $S\left(\mathfrak{h}_{\ell}\right)^{W_{\ell}}=$ $\mathbb{C}\left[\epsilon_{1}, \ldots, \epsilon_{\ell}\right]^{W_{\ell}}(\underline{\operatorname{Lus}})$.

Define elements $y_{i} \in \mathcal{H}_{\ell}(i=1, \ldots, \ell)$ by

$$
y_{i}:=s_{1 i} \cdot \epsilon_{i} \cdot s_{1 i}=\epsilon_{i}-\sum_{j<i} s_{j i} .
$$


Then one can see by direct calculations that

$$
\begin{aligned}
& w \cdot y_{i}=y_{w(i)} \cdot w \quad\left(i=1, \ldots, \ell, w \in W_{\ell}\right), \\
& {\left[y_{i}, y_{j}\right]=-\left(y_{i}-y_{j}\right) s_{i j} \quad(1 \leq i, j \leq \ell) .}
\end{aligned}
$$

The algebra $\mathcal{H}_{\ell}$ is isomorphic to the $\mathbb{C}$-algebra with generators $w \in W_{\ell}$ and $y_{i}$ $(i=1, \ldots, \ell)$ with the defining relations (1.3.3), (1.3.4) and the Coxeter relations of $w$ 's in $W_{\ell}$.

Let

$$
F_{i} \mathcal{H}_{\ell}:=\sum_{w \in W_{\ell}} \sum_{d_{1}+d_{2}+\cdots+d_{\ell} \leq i} \mathbb{C} y_{1}^{d_{1}} y_{2}^{d_{2}} \ldots y_{\ell}^{d_{\ell}} w \quad\left(i \in \mathbb{Z}_{\geq 0}\right)
$$

Then, by (1.3.3), $F_{i} \mathcal{H}_{\ell} \cdot F_{j} \mathcal{H}_{\ell} \subset F_{i+j} \mathcal{H}_{\ell}$, and 1.3.5 defines a filteration on $\mathcal{H}_{\ell}$. Let gr $\mathcal{H}_{\ell}$ denote the corresponding graded algebra;

$$
\operatorname{gr} \mathcal{H}_{\ell}=\bigoplus_{i \in \mathbb{Z}_{\geq 0}} F_{i} \mathcal{H}_{\ell} / F_{i-1} \mathcal{H}_{\ell} \quad\left(F_{-1} \mathcal{H}_{\ell}=0\right)
$$

Let $\bar{y}_{i}$ denote the image of $y_{i}$ in gr $\mathcal{H}_{\ell}$. Then gr $\mathcal{H}_{\ell}$ is isomorphic to the graded $\mathbb{C}$ algebra generated by $\mathbb{C}\left[W_{\ell}\right]$ and the polynomial ring $\mathbb{C}\left[\bar{y}_{1}, \ldots, \bar{y}_{\ell}\right]$ with the relations $w \cdot \bar{y}_{i}=\bar{y}_{w(i)} \cdot w\left(i=1, \ldots, \ell, w \in W_{\ell}\right)$, whose grading is given by $\operatorname{deg}\left(y_{i}\right)=1$ and $\operatorname{deg}(w)=0$. In particular, gr $\mathcal{H}_{\ell} \cong \mathbb{C}\left[\bar{y}_{1}, \ldots, \bar{y}_{\ell}\right] \otimes_{\mathbb{C}} \mathbb{C}\left[W_{\ell}\right]$ as a $\mathbb{C}$-vector space.

1.4. Representations of degenerate affine Hecke algebra. In this subsection we review the theory of the representations of $\mathcal{H}_{\ell}$ studied in [Ze], Ro, Gin] (see also [CG], along the line introduced in [AS] and developed in $\mathrm{S}$.

Let $\mathcal{C}_{\mathcal{H}_{\ell}}$ denote the category of finite-dimensional representations of $\mathcal{H}_{\ell}$. Let $r$ be a nonnegative integer. The representation theory of $\mathcal{H}_{\ell}$ is well-described by the language of the Bernstein-Gelfand-Gelfand category $\mathcal{O}_{r}$ of $\mathfrak{g l}_{r}$, via the functors $F_{\lambda}: \mathcal{O}_{r} \rightarrow \mathcal{C}_{\mathcal{H}_{\ell}}$ constructed in $\mathrm{AS}, \mathrm{S}$ :

Let

$$
\begin{aligned}
P_{r}^{+} & :=\left\{\lambda \in \mathfrak{h}_{r}^{*} \mid \lambda(\alpha) \notin-1,-2, \ldots \text { for all } \alpha \in R_{r}^{+}\right\}, \\
P_{r, \mathbb{Z}}^{+} & :=\bigoplus_{i=1}^{r} \mathbb{Z} \epsilon_{i} \subset P_{r}^{+} .
\end{aligned}
$$

An element of $P_{r}^{+}$(resp. $P_{r, \mathbb{Z}}^{+}$) is called the dominant (resp. integral dominant) weight.

For $\lambda \in \mathfrak{h}_{r}^{*}$, there is a functor form $\mathcal{O}_{r}$ to $\mathcal{C}_{\mathcal{H}_{\ell}}$ defined by

$$
\begin{aligned}
F_{\lambda}(X): & =H_{0}\left(\mathfrak{n}_{r}^{-}, X \otimes\left(\mathbb{C}^{r}\right)^{\otimes \ell}\right)_{\lambda-\rho} \quad\left(X \in \mathcal{O}_{r}\right) \\
& =\left[X \otimes\left(\mathbb{C}^{r}\right)^{\otimes \ell} / \mathfrak{n}_{r}^{-}\left(X \otimes\left(\mathbb{C}^{r}\right)^{\otimes \ell}\right)\right]_{\lambda-\rho},
\end{aligned}
$$

where $\mathfrak{n}_{r}^{-}$denotes the nilpotent subalgebra of $\mathfrak{g l}_{r}$ generated by the lower triangular matrices $\left\langle E_{i j} \mid i-j>0\right\rangle$ and $X_{\lambda}$ denotes the weight space of weight $\lambda$ of a $\mathfrak{g l}_{r^{-}}$ module $X$. The action of $\mathcal{H}_{\ell}$ on the space $F_{\lambda}(X)$ is given by

$$
\begin{aligned}
& \epsilon_{i} \longmapsto \sum_{j=0}^{i} \Omega_{j i}+\frac{r-1}{2} \quad(1 \leq i \leq \ell) \\
& s_{i} \longmapsto \Omega_{i i+1} \quad(1 \leq i \leq \ell-1),
\end{aligned}
$$

where $\Omega_{i j}$ denotes an endomorphism of $X \otimes\left(\mathbb{C}^{r}\right)^{\otimes \ell}$ which acts as the Casimir $\Omega=\sum_{r s} E_{r s} \otimes E_{s r}$ on the tensor product of $i$-th and $j$-th factors and by identity 
on all the other factors. Here the 0-th factor corresponds to $X \in \mathcal{O}_{r}$. The functor $F_{\lambda}$ is exact if $\lambda \in P_{r}^{+}($AS $)$.

For complex numbers $a, b$ such that $b-a+1=\ell$, let $\mathbb{C}_{[a, b]}=\mathbb{C} \mathbf{1}_{[a, b]}$ denote the one-dimensional representation of $\mathcal{H}_{\ell}$, defined by

$$
\begin{array}{r}
s_{i} \cdot \mathbf{1}_{[a, b]}=\mathbf{1}_{[a, b]} \quad(i=1, \ldots, \ell-1), \\
\epsilon_{i} \cdot \mathbf{1}_{[a, b]}=(a+i-1) \mathbf{1}_{[a, b]} \quad(i=1, \ldots, \ell) .
\end{array}
$$

Let $\operatorname{Wt}(X)$ denote the space of weights of a $\mathfrak{g l}_{r}$-module $X$. Then, clearly

$$
\left.\mathrm{Wt}\left(\left(\mathbb{C}^{r}\right)^{\otimes \ell}\right)\right)=\left\{\sum_{i=1}^{r} n_{i} \epsilon_{i} \mid n_{i} \in\{0,1, \ldots, \ell\}, \sum_{i=1}^{r} n_{i}=\ell\right\} .
$$

For $\lambda \in \mathfrak{h}_{r}^{*}$, let

$$
\left.S(\lambda ; \ell):=\left\{\mu \in \mathfrak{h}_{r}^{*} \mid \lambda-\mu \in \mathrm{Wt}\left(\left(\mathbb{C}^{r}\right)^{\otimes \ell}\right)\right)\right\} .
$$

For $\lambda \in \mathfrak{h}_{r}^{*}$ and $\mu \in S(\lambda, \ell)$, define an $\mathcal{H}_{\ell}$-module

$$
\mathcal{K}(\lambda, \mu):=\mathcal{H}_{\ell} \otimes\left(\mathcal{H}_{\ell_{1}} \otimes \ldots \otimes \mathcal{H}_{\ell_{r}}\right)\left(\mathbb{C}_{\left[\mu_{1}, \lambda_{1}-1\right]} \otimes \ldots \otimes \mathbb{C}_{\left[\mu_{r}, \lambda_{r}-1\right]}\right)
$$

where $\lambda_{i}=\lambda\left(\epsilon_{i}\right), \mu_{i}=\mu\left(\epsilon_{i}\right), \ell_{i}=\lambda_{i}-\mu_{i}$. Here $\mathcal{H}_{\ell_{1}} \otimes \mathcal{H}_{\ell_{2}} \otimes \ldots \otimes \mathcal{H}_{\ell_{r}}$ is regarded as a subalgebra of $\mathcal{H}_{\ell}$ via the embeddings $\mathcal{H}_{\ell_{k}} \hookrightarrow \mathcal{H}_{\ell}$ defined by $\epsilon_{a} \mapsto \epsilon_{a+\sum_{j=1}^{k-1} \ell_{j}}$ and $s_{a} \mapsto s_{a+\sum_{j=1}^{k-1} \ell_{j}}$.

Let

$$
\mathbf{1}_{\lambda, \mu}:=\mathbf{1}_{\left[\mu_{1}, \lambda_{1}-1\right]} \otimes \ldots \otimes \mathbf{1}_{\left[\mu_{r}, \lambda_{r}-1\right]} \in \mathcal{K}(\lambda, \mu) .
$$

Then the correspondence $\mathbf{1}_{\lambda, \mu} \mapsto 1$ defines an isomorphism of $W_{\ell^{-}}$-modules

$$
\mathcal{K}(\lambda, \mu) \cong \mathbb{C}\left[W_{\ell} / W_{\ell_{1}} \times \cdots \times W_{\ell_{r}}\right] .
$$

For a partition $\nu$ of $\ell$, let $U(\nu)$ denote the simple $W_{\ell}$-module associated with $\nu$. For $\lambda \in \mathfrak{h}_{r}^{*}$ and $\mu \in S(\lambda ; \ell)$, let $\nu_{\lambda, \mu}$ denote the partition of $\ell$ obtained by forgetting the order of $\left(\ell_{1}, \ldots, \ell_{\ell}\right)$, where $\ell_{i}=(\lambda-\mu)\left(\epsilon_{i}\right)$. Then by 1.4.6), $\mathcal{K}(\lambda, \mu)$ decomposes as

$$
\mathcal{K}(\lambda, \mu) \cong U\left(\nu_{\lambda, \nu}\right) \oplus \bigoplus_{\nu>\nu_{\lambda, \nu}} U(\nu)^{\oplus c_{\nu}}
$$

as a $W_{\ell}$-module, where $>$ is the dominance order in the set of the partitions and $c_{\nu}$ is some nonnegative integer (see [FH], for example). It is known that if $\lambda \in P_{r}^{+}$, the $W_{\ell}$-simple component $U\left(\nu_{\lambda, \mu}\right)$ generates $\mathcal{K}(\lambda, \mu)$ over $\mathcal{H}_{\ell}$, hence it has an unique simple quotient $\mathcal{L}(\lambda, \mu)$ which contains $U\left(\nu_{\lambda, \mu}\right)$ with multiplicity one (Ze], R0], see also [S]). The module $\mathcal{K}(\lambda, \mu)$ with $\lambda \in P_{r}^{+}$and $\mu \in S(\lambda ; \ell)$ is called a standard module of $\mathcal{H}_{\ell}$.

Let $W_{\lambda} \subset W_{r}$ denote the stabilizer of $\lambda \in \mathfrak{h}_{r}^{*}$. Notice that if $\mu \in S(\lambda ; \ell)$, then $w \cdot \mu \in S(\lambda ; \ell)$ for all $w \in W_{\lambda}$. One has

$$
\begin{aligned}
\mathcal{K}(\lambda, \mu) \cong \mathcal{K}\left(\lambda, \mu^{\prime}\right) & \Longleftrightarrow \quad \mathcal{L}(\lambda, \mu) \cong \mathcal{L}\left(\lambda, \mu^{\prime}\right) \\
& \Longleftrightarrow \mu^{\prime}=w \cdot \mu \text { for some } w \in W_{\lambda}
\end{aligned}
$$

for $\lambda \in P_{r}^{+}$and $\mu, \mu^{\prime} \in S(\lambda ; \ell)$ (Ze], Rd]). It is known that any simple $\mathcal{H}_{\ell}$-module is isomorphic to $\mathcal{L}(\lambda, w \cdot \mu)$ for some $\lambda, \mu \in P_{r}^{+}$and $w \in W_{\lambda} \backslash W_{r} / W_{\mu}$ such that $w \cdot \mu \in S(\lambda ; \ell)$ for some $r \in \mathbb{N}$. ([Z], $\mathrm{Ro})$. 
Let $M(\lambda)$ be the Verma module of $\mathfrak{g l}_{r}$ with highest weight $\lambda-\rho$ and let $L(\lambda)$ denote its unique simple quotient. Let $\lambda, \mu \in P_{r}^{+}$and $w \in W_{\lambda} \backslash W_{r} / W_{\mu}$ such that $w \cdot \mu \in S(\lambda ; \ell)$. Then one of the main results in [AS, S] is stated as follows:

$$
\begin{aligned}
& F_{\lambda}(M(w \cdot \mu)) \cong \mathcal{K}(\lambda, w \cdot \mu), \\
& F_{\lambda}(L(w \cdot \mu)) \cong \begin{cases}\mathcal{L}(\lambda, w \cdot \mu) & \text { if } w \cdot \mu=w_{L R} \cdot \mu, \\
0 & \text { otherwise }\end{cases}
\end{aligned}
$$

where $w_{L R}$ denotes the longest length representative of $w$ in the double coset $W_{\lambda} w W_{\mu}$.

\section{Drinfeld FunCtor}

2.1. Drinfeld Functor. For a left $\mathcal{H}_{\ell}$-module $M$, consider an $\mathcal{H}_{\ell} \otimes U\left(\mathfrak{g l}_{n}\right)$-module $M \otimes\left(\mathbb{C}^{n}\right)^{\otimes \ell}$. Here we regard $\mathbb{C}^{n}$ as the vector representation of $\mathfrak{g l}_{n}$. For $x \in \mathfrak{g l}_{n}$ and $i=1, \ldots, \ell$, let $\tau_{i}(x)$ denote the endomorphism of $M \otimes\left(\mathbb{C}^{n}\right)^{\otimes \ell}$ which acts as $x \in \mathfrak{g l}_{n}$ on the $i$-th factor of the tensor product $\left(\mathbb{C}^{n}\right)^{\otimes \ell}$ and by identity on all the other factors.

Define an action of the Yangian $Y\left(\mathfrak{g l}_{n}\right)$ on $M \otimes\left(\mathbb{C}^{n}\right)^{\otimes \ell}$ by

$$
\pi: t(u) \longmapsto T_{1}\left(u-\epsilon_{1}\right) T_{2}\left(u-\epsilon_{2}\right) \ldots T_{\ell}\left(u-\epsilon_{\ell}\right),
$$

where

$$
T_{i}\left(u-\epsilon_{i}\right)=1+\frac{1}{u-\epsilon_{i}} I_{i}
$$

and $I_{i}=\sum_{1 \leq a, b \leq n} \tau_{i}\left(E_{a b}\right) \otimes E_{a b} \in \operatorname{End}\left(M \otimes\left(\mathbb{C}^{n}\right)^{\otimes \ell}\right) \otimes \operatorname{End}\left(\mathbb{C}^{n}\right)$. By the fact that $S\left(\mathfrak{h}_{\ell}\right)$ is commutative, 2.1.1) gives a well-defined action of $Y\left(\mathfrak{g l}_{n}\right)$ (recall (1.1.3) and (1.1.4)).

The symmetric group $W_{\ell}$ naturally acts on $M \otimes\left(\mathbb{C}^{n}\right)^{\otimes \ell}$ by $s_{i j} \mapsto K_{i j} P_{i j}$, where $K_{i j}$ denotes its action on $M$ and $P_{i j}$ denotes its action on $\left(\mathbb{C}^{n}\right)^{\otimes \ell}$ by permutation. Now define

$$
D_{\ell}(M):=\left(M \otimes\left(\mathbb{C}^{n}\right)^{\otimes \ell}\right) / \sum_{i=1}^{\ell} \operatorname{Im}\left(s_{i}+1\right),
$$

where $\operatorname{Im}\left(s_{i}+1\right)$ denotes the subspace $\left(s_{i}+1\right)\left(M \otimes\left(\mathbb{C}^{n}\right)^{\otimes \ell}\right)$. Let $[m \otimes u]$ denote the equivalence class of $m \otimes u \in M \otimes\left(\mathbb{C}^{n}\right)^{\otimes \ell}$ in $D_{\ell}(M)$.

The following proposition is due to Drinfeld ([D2], see also BGHP, CP2 ).

Proposition 2. Let $M$ be an $\mathcal{H}_{\ell}$-module. Then, $\pi$ induces an action of $Y\left(\mathfrak{g l}_{n}\right)$ on the space $D_{\ell}(M)$.

Proof. It is enough to show that $\left(\prod_{i=1}^{\ell}\left(u-\epsilon_{i}\right)\right) \cdot \pi(t(u))$ preserves the denominator space of 2.1.2) since $\left(\prod_{i=1}^{\ell}\left(u-\epsilon_{i}\right)\right) \in Z\left(\mathcal{H}_{\ell}\right)[u]$. This follows from the formula

$$
\begin{aligned}
& \left(u-\epsilon_{i}+I_{i}\right)\left(u-\epsilon_{i+1}+I_{i+1}\right) s_{i} \\
& \equiv s_{i}\left(u-\epsilon_{i}+I_{i}\right)\left(u-\epsilon_{i+1}+I_{i+1}\right)+\left(s_{i}+1\right)\left[I_{i+1}, I_{i}\right]
\end{aligned}
$$

$\left(s_{i}=K_{i i+1} P_{i i+1}\right)$, which can be proven by direct calculations using the defining relations (1.3.1) and the commutation relations $\left[P_{i}, I_{i}\right]=\left[I_{i+1}, I_{i}\right]$. 
The action of $Y\left(\mathfrak{g l}_{n}\right)$ on the space $D_{\ell}(M)$ will be denoted by the same symbol $\pi$.

Let $\mathcal{C}_{Y\left(\mathfrak{g l}_{n}\right)}$ and $\mathcal{C}_{Y\left(\mathfrak{s} \mathfrak{l}_{n}\right)}$ denote the category of finite-dimensional representations of $Y\left(\mathfrak{g l}_{n}\right)$ and $Y\left(\mathfrak{s l}_{n}\right)$ respectively. Then $D_{\ell}$ defines an exact functor from $\mathcal{C}_{\mathcal{H}_{\ell}}$ to $\mathcal{C}_{Y\left(\mathfrak{g l}_{n}\right)}$ or $\mathcal{C}_{Y\left(\mathfrak{s l}_{n}\right)}$. The functor $D_{\ell}$ is called the Drinfeld Functor ([D2]). Note that our definition of the Drinfeld functor slightly differs from that of [D2]. A thorem of Drinfeld states that if $\ell<n, D_{\ell}$ gives a categorical equivalence between $\mathcal{C}_{\mathcal{H}_{\ell}}$ and the certain subcategory of $\mathcal{C}_{Y\left(\mathfrak{s l}_{n}\right)}$ ([D2]). One can deduce its unpublished proof from the paper of Chari-Pressly [CP2], in which the categorical equivalnece was generalized to the case between the affine Hecke algebra and the quantum affine algebra. However, the method in [CP2] does not apply to the case $\ell \geq n$.

2.2. The following proposition follows from the Frobenius-Schur duality.

Proposition 3. Let $M$ be an $\mathcal{H}_{\ell}$-module. Let $M=\bigoplus_{\nu} U(\nu)^{\oplus c_{\nu}}\left(c_{\nu} \in \mathbb{Z}_{\geq 0}\right)$ be a decomposition of $M$ as a $W_{\ell}$-module. Then,

$$
D_{\ell}(M) \cong \bigoplus_{\nu\left(\epsilon_{1}\right) \leq n} L\left(\nu^{\prime}+\rho\right)^{\oplus c_{\nu}}
$$

as a $\mathfrak{g l}_{n}$-module, where $\nu^{\prime}$ is the transpose of a partition $\nu$ identified with the dominant integral weight of $\mathfrak{g l}_{n}$.

See [CP2] for the proof of the following proposition.

Proposition 4. Let $M_{1}$ and $M_{2}$ be representations of $\mathcal{H}_{\ell_{1}}$ and $\mathcal{H}_{\ell_{2}}$ respectively. Then,

$$
D_{\ell_{1}}\left(M_{1}\right) \otimes D_{\ell_{2}}\left(M_{2}\right) \cong D_{\ell_{1}+\ell_{2}}\left(\mathcal{H}_{\ell_{1}+\ell_{2}} \otimes_{\left(\mathcal{H}_{\ell_{1}} \otimes \mathcal{H}_{\ell_{2}}\right)}\left(M_{1} \otimes M_{2}\right)\right)
$$

as a $Y\left(\mathfrak{g l}_{n}\right)$ and $Y\left(\mathfrak{s l}_{n}\right)$-module.

The following formula was stated in $[\overline{B G H P}]$ as a conjecture.

Proposition 5. Let $M$ be an $\mathcal{H}_{\ell}$-module. Then,

$$
\pi\left(t_{a b}(u)\right) \equiv \delta_{a b}+\sum_{i=1}^{\ell} \frac{1}{u-y_{i}} \otimes \tau_{i}\left(E_{a b}\right)
$$

on the space $D_{\ell}(M)$. In particular, $\pi\left(t_{a b}^{(d)}\right)$ acts as $\sum_{i=1}^{\ell} y_{i}^{d} \otimes \tau_{i}\left(E_{a b}\right)$.

Proof. We prove by induction on $k$ that

$$
\left(1+\frac{I_{1}}{u-\epsilon_{1}}\right)\left(1+\frac{I_{2}}{u-\epsilon_{2}}\right) \ldots\left(1+\frac{I_{k}}{u-\epsilon_{k}}\right) \equiv\left(1+\sum_{i=1}^{k} \frac{1}{u-y_{i}} I_{i}\right)
$$

on $D_{\ell}(M)$.

There is nothing to prove for $k=1$. Let $k>1$. By induction hypothesis,

$$
\begin{aligned}
& \left(1+\frac{I_{1}}{u-\epsilon_{1}}\right)\left(1+\frac{I_{2}}{u-\epsilon_{2}}\right) \ldots\left(1+\frac{I_{k}}{u-\epsilon_{k}}\right) \\
& \equiv 1+\left(\sum_{i=1}^{k-1} \frac{1}{u-y_{i}} I_{i}\right)+\frac{1}{u-\epsilon_{k}} I_{k}+\left(\sum_{i=1}^{k-1} \frac{1}{u-y_{i}}\right) \cdot \frac{1}{u-\epsilon_{k}} I_{i} I_{k}
\end{aligned}
$$


Since $I_{i} \cdot I_{k}=P_{i k} \cdot I_{k}$ and $P_{i k} \equiv-K_{i k}$ on $D_{\ell}(M)$,

$$
\begin{aligned}
& \frac{1}{u-\epsilon_{k}} I_{k}+\left(\sum_{i=1}^{k-1} \frac{1}{u-y_{i}}\right) \cdot \frac{1}{u-\epsilon_{k}} I_{i} I_{k} \\
& \equiv\left(\frac{1}{u-\epsilon_{k}}-\sum_{i=1}^{k-1} K_{i k} \cdot \frac{1}{u-y_{i}} \cdot \frac{1}{u-\epsilon_{k}}\right) I_{k} \\
& =\frac{1}{u-y_{k}}\left(u-y_{k}-\sum_{i=1}^{k-1} K_{i k}\right) \frac{1}{u-\epsilon_{k}} I_{k} \\
& =\frac{1}{u-y_{k}} \cdot\left(u-\epsilon_{k}\right) \cdot \frac{1}{u-\epsilon_{k}} I_{k}=\frac{1}{u-y_{k}} I_{k} .
\end{aligned}
$$

Let $\Lambda_{k}=\sum_{i=1}^{k} \epsilon_{i} \in P_{n}^{+}$for $k=0, \ldots, n$ and let $v_{\Lambda_{k}}$ denote the highest weight vector of the simple $\mathfrak{g l}_{n}$-module $L\left(\Lambda_{k}+\rho\right)$. Then we can identify $v_{\Lambda_{k}}$ with the highest weight vector of the simple $Y\left(\mathfrak{g l}_{n}\right)$-module ev $a\left(L\left(\Lambda_{k}+\rho\right)\right)(a \in \mathbb{C})$. It can be checked directly that its weight $\left(\zeta_{1}(u), \ldots, \zeta_{n}(u)\right)$ is given by

$$
\zeta_{i}(u)= \begin{cases}1+\frac{1}{u-i-a} & \text { if } 1 \leq i \leq k \\ 1 & \text { otherwise }\end{cases}
$$

The following proposition, which is easily follows from Proposition 5, is due to Chari-Pressley [CP2].

Proposition 6. Let $a, b$ be complex numbers such that $b-a+1=\ell$. Then, as $a$ $Y\left(\mathfrak{g l}_{n}\right)$-module,

$$
D_{\ell}\left(\mathbb{C}_{[a, b]}\right) \cong \begin{cases}\operatorname{ev}_{a}\left(L\left(\Lambda_{\ell}+\rho\right)\right) & \text { if } \ell \leq n \\ 0 & \text { otherwise }\end{cases}
$$

\section{MAin Results}

3.1. For a subspace $M^{\prime}$ of an $\mathcal{H}_{\ell}$-module $M$, let $D_{\ell}\left(M^{\prime}\right)$ denote the image of $M^{\prime}$ by the Drinfeld functor in $D_{\ell}(M)$. The proof of the following proposition is in Section 1

Proposition 7. Let $M$ be an $\mathcal{H}_{\ell}$-module such that $M$ is generated by some simple $W_{\ell}$-submodule $U$ of $M$. Suppose that $D_{\ell}(U)$ is nonzero. Then, $D_{\ell}(U)$ generates $D_{\ell}(M)$ over $Y\left(\mathfrak{g l}_{n}\right)$.

Now let $r \in \mathbb{N}$. For $\lambda \in \mathfrak{h}_{r}^{*}$, let

$$
S^{(n)}(\lambda)=\left\{\mu \in \mathfrak{h}_{r} \mid(\lambda-\mu)\left(\epsilon_{i}\right) \in\{0,1, \ldots, n\} \text { for } i=1, \ldots, r\right\} .
$$

For $\lambda \in \mathfrak{h}_{r}^{*}$ and $\mu \in S^{(n)}(\lambda)$, define a tensor product module $M(\lambda, \mu)$ of $Y\left(\mathfrak{g l}_{n}\right)$ by

$$
M(\lambda, \mu):=\operatorname{ev}_{\mu_{1}}\left(L\left(\Lambda_{\ell_{1}}+\rho\right)\right) \otimes \ldots \otimes \operatorname{ev}_{\mu_{r}}\left(L\left(\Lambda_{\ell_{r}}+\rho\right)\right),
$$

where $\mu_{i}=\mu\left(\epsilon_{i}\right), \ell_{i}=(\lambda-\mu)\left(\epsilon_{i}\right)$. Here $Y\left(\mathfrak{g l}_{n}\right)$ acts via the coproduct (1.1.3). Let $v_{\lambda, \mu}:=v_{\Lambda_{\ell_{1}}} \otimes \ldots \otimes v_{\Lambda_{\ell_{r}}} \in M(\lambda, \mu)$. Then by (2.2.2), $t_{i i}(u) \cdot v_{\lambda, \mu}=\zeta_{\lambda, \mu ; i}(u) v_{\lambda, \mu}$ for 
$i=1, \ldots, n$, where

$$
\zeta_{\lambda, \mu ; i}(u)=\prod_{\substack{j=1, \ldots, \ell \\ \ell_{j} \geq i}}\left(1+\frac{1}{u-i-\mu_{j}}\right) .
$$

Let $S^{(n)}(\lambda ; \ell)=S(\lambda ; \ell) \cap S^{(n)}(\lambda)$. Notice that for $\mu \in S(\lambda ; \ell)$, the condition $\mu \in S^{(n)}(\lambda ; \ell)$ is equivalent to $\nu_{\lambda, \mu}\left(\epsilon_{1}\right) \leq n$, where the partition $\nu_{\lambda, \mu}$ is identified with a dominant integral weight (recall subsection 1.4).

\section{Theorem 8.}

(1) The Drinfeld functor sends a standard module of $\mathcal{H}_{\ell}$ to zero or a highest weight module of $Y\left(\mathfrak{g l}_{n}\right)$.

(2) More precisely, let $\lambda \in P_{r}^{+}$and $\mu \in S(\lambda ; \ell)$. Then,

$$
D_{\ell}(\mathcal{K}(\lambda, \mu)) \cong \begin{cases}M(\lambda, \mu) & \text { if } \mu \in S^{(n)}(\lambda ; \ell), \\ 0 & \text { otherwise. }\end{cases}
$$

In particular, $M(\lambda, \mu)$ is highest weight with the highest weight vector $v_{\lambda, \mu}$ and the highest weight $\zeta_{\lambda, \mu}(u)$.

Proof. (1) Let $M$ be a standard module and suppose that $D_{\ell}(M) \neq 0$. Since $M$ is a standard module, $M \cong U(\nu) \oplus \underset{\substack{\gamma>\nu \\|\gamma|=\ell}}{\bigoplus} U(\gamma)^{\oplus c_{\gamma}}$ and $M=\mathcal{H}_{\ell} \cdot U(\nu)$ for some partition $\nu$ of $\ell$ such that $\nu\left(\epsilon_{1}\right) \leq n$. By Proposition 3 ,

$$
D_{\ell}(M) \cong L\left(\nu^{\prime}+\rho\right) \oplus \bigoplus_{\substack{\gamma^{\prime}<\nu^{\prime}, \gamma\left(\epsilon_{1}\right) \leq n \\|\gamma|=\ell}} L\left(\gamma^{\prime}+\rho\right)^{\oplus c_{\gamma}}
$$

But by Proposition 7 , the highest weight vector of $L\left(\nu^{\prime}+\rho\right)$ generates $D_{\ell}(M)$ over $Y\left(\mathfrak{g l}_{n}\right)$ in this decomposition. Since the other $\mathfrak{g l}_{n}$-weights appearing in $D_{\ell}(M)$ is smaller than $\nu^{\prime}$ with respect to the dominance order, it follows that $D_{\ell}(M)$ is a highest weight module whose highest weight vector is the $\mathfrak{g l}_{n}$-highest weight vector of $L\left(\nu^{\prime}+\rho\right) \subset D_{\ell}(M)$. (2) The isomorphism (3.1.3) follows from Proposition 4 and Proposition 6. In fact, (3.1.3) holds without restriction $\lambda \in P_{\ell}^{+}$. The rest of the statement follows from (1).

Remark 9. The fact that $M(\lambda, \mu)$ is highest weight for $\lambda \in P_{r}^{+}$was proved by Akasaka-Kashiwara ( AK $)$ in the case of the quantum affine algebra and by NazarovTarasov ( $\sqrt{\mathrm{NT} 2}]$ ) in the case of the Yangian. The above thorem provides another proof of it.

Let us call those $Y\left(\mathfrak{g l}_{n}\right)$-modules $M(\lambda, \mu)$ with $\lambda \in P_{r}^{+}$and $\mu \in S^{(n)}(\lambda ; \ell)$ standard tensor product modules of $Y\left(\mathfrak{g l}_{n}\right)$. By Theorem 8 (2), a standard tensor product module $M(\lambda, \mu)$ has a unique simple quotient, which is denoted by $V(\lambda, \mu)$. Then by (3.1.2), its Drinfeld polynomials $Q_{\lambda, \mu}(u)=\left(Q_{\lambda, \mu ; 1}(u), \ldots, Q_{\lambda, \mu ; n-1}(u)\right)$ are caluculated as

$$
Q_{\lambda, \mu ; k}(u)=\prod_{\substack{i=1, \ldots, r \\ \lambda_{i}-\mu_{i}=k}}\left(u-\lambda_{i}\right),
$$

where $\lambda_{i}=\lambda\left(\epsilon_{i}\right)$ and $\mu_{i}=\mu\left(\epsilon_{i}\right)$ ([AK, CP2, NT2]). 
DRINFELD FUNCTOR AND FINITE-DIMENSIONAL REPRESENTATIONS OF YANGIAN 11

\section{Theorem 10.}

(1) The Drinfeld functor sends a simple $\mathcal{H}_{\ell}$-module to zero or a simple $Y\left(\mathfrak{g l}_{n}\right)$ module.

(2) More precisely, let $\lambda \in P_{r}^{+}$and $\mu \in S(\lambda ; \ell)$. Then,

$$
D_{\ell}(\mathcal{L}(\lambda, \mu)) \cong \begin{cases}V(\lambda, \mu) & \text { if } \mu \in S^{(n)}(\lambda ; \ell), \\ 0 & \text { otherwise. }\end{cases}
$$

Proof. (1) Let $\mathcal{L}$ be a simple $\mathcal{H}_{\ell}$-module. Suppose that $D_{\ell}(\mathcal{L}) \neq 0$. Let $V$ be a proper $Y\left(\mathfrak{g l}_{n}\right)$-submodule of $D_{\ell}(\mathcal{L})$. We suppose that $V \neq 0$ and deduce a contradiction. Let $L$ be a simple $\mathfrak{g l}_{n}$-submodule of $V$. Then $L=D_{\ell}(U)$ for some simple $W_{\ell}$-submodule $U$ of $M$. But since $M$ is simple, $M=\mathcal{H}_{\ell} \cdot U$. Thus by Proposition 7. $D_{\ell}(M)=Y\left(\mathfrak{g l}_{n}\right) \cdot L \subset Y\left(\mathfrak{g l}_{n}\right) \cdot V$, which contradicts the assumption that $V$ is proper. (2) follows from (1), Theorem $8(2)$ and and the fact that $\mathcal{L}(\lambda, \mu)$ contains the simple $W_{\ell}$-module $U\left(\nu_{\lambda, \mu}\right)$ with multiplicity one.

Remark 11. By (3.1.4), it is easy to see that every simple $Y\left(\mathfrak{s l}_{n}\right)$-module appears as the image of a simple $\mathcal{H}_{\ell}$-module by the Drinfeld functor for some $\ell$.

Remark 12. When $\lambda-\rho$ and $\mu-\rho$ are both dominant weights, $V(\lambda, \mu)$ belongs to the class of representations called tame (NT1). Conversely, any simple tame module is isomorphic to $V(\lambda, \mu)$ with $\lambda-\rho, \mu-\rho \in P_{r}^{+}$for some $\ell$.

3.2. Multiplicity Formula. For $\lambda, \mu \in P_{r}^{+}$, define

$$
W_{r}^{(n)}(\lambda, \mu)=\left\{w \in W_{r} \mid w \cdot \mu \in S^{(n)}(\lambda ; \ell)\right\} \subset W_{r} .
$$

Then $S^{(n)}(\lambda ; \ell)=\bigsqcup_{\mu \in P_{r}^{+}} \bigcup_{w \in W_{r}^{(n)}(\lambda, \mu)}\{w \cdot \mu\}$. Notice that if $\mu \in S^{(n)}(\lambda ; \ell)$, then $w \cdot \mu \in S^{(n)}(\lambda ; \ell)$ for all $w \in W_{\lambda}$.

Lemma 13. Let $\lambda, \mu \in P_{r}^{+}$and $w, w^{\prime} \in W_{r}^{(n)}(\lambda, \mu)$. Then $Q_{\lambda, w \cdot \mu}(u)=Q_{\lambda, w^{\prime} \cdot \mu}(u)$ if and only if $w \equiv w^{\prime}$ in the double coset $W_{\lambda} \backslash W_{r} / W_{\mu}$.

Proof. First notice that the condition $w \equiv w^{\prime}$ in the double coset $W_{\lambda} \backslash W_{r} / W_{\mu}$ is equivalent to the condition that the following sets of pairs of complex numbers are equal;

$$
\left\{\left(\lambda\left(\epsilon_{i}\right), w \cdot \mu\left(\epsilon_{i}\right)\right) \mid i=1, \ldots, r\right\}, \quad\left\{\left(\lambda\left(\epsilon_{i}\right), w^{\prime} \cdot \mu\left(\epsilon_{i}\right)\right) \mid i=1, \ldots, r\right\} .
$$

Hence the direction $\Leftarrow$ is easy to see. $\Rightarrow$. Let

$$
\left\{\left(a_{1}, b_{1}\right), \cdots,\left(a_{k}, b_{k}\right)\right\}, \quad\left\{\left(a_{1}, b_{1}^{\prime}\right), \cdots,\left(a_{k}, b_{k}^{\prime}\right)\right\}
$$

be the result of removing the common pairs from (3.2.1) so that $a_{i} \geq a_{j}$ if $a_{i}-a_{j} \in \mathbb{Z}$ for $1 \leq i<j \leq k$. We suppose that $k \geq 1$ and deduce a contradiction. By the assumption $Q_{\lambda, w \cdot \mu}(u)=Q_{\lambda, w^{\prime} \cdot \mu}(u)$, the differences $a_{i}-b_{i}$ and $a_{i}-b_{i}^{\prime}$ are all 0 or $n$.

Now by the assumption, $b_{1} \neq b_{1}^{\prime}$. We can assume $a_{1}=b_{1}+n=b_{1}^{\prime}$. Since $\left\{b_{i}\right\}_{i=1}^{k}=\left\{b_{i}^{\prime}\right\}_{i=1}^{k}$, there exists $p$ such that $b_{p}=b_{1}^{\prime}$. Then $a_{p}=b_{p}\left(=a_{1}\right)$ since $a_{p}=b_{p}$ or $b_{p}+n$ and $a_{1} \geq a_{p}$. Hence $\left(a_{1}^{\prime}, b_{1}\right)=\left(a_{p}, b_{p}\right)$, which contradicts the assumption that there is no common pair between the two sets in (3.2.2). 
Proposition 14. Let $\lambda, \mu \in P_{r}^{+}$and $w, w^{\prime} \in W_{r}^{(n)}(\lambda, \mu)$. Then the following conditions are equivalent:

(1) $M(\lambda, w \cdot \mu) \cong M\left(\lambda, w^{\prime} \cdot \mu\right)$.

(2) $V(\lambda, w \cdot \mu) \cong V\left(\lambda, w^{\prime} \cdot \mu\right)$.

(3) $w \equiv w^{\prime}$ in the double coset $W_{\lambda} \backslash W_{r} / W_{\mu}$.

Proof. (1) $\Rightarrow(2)$ follows from Theorem 8 and the fact that a simple quotient of a highest weight module is unique. (2) $\Rightarrow$ (3) follows from Lemma 13 and $(3) \Rightarrow$ (1) follows from (1.4.8).

Let $\overline{W_{r}^{(n)}(\lambda, \mu)}$ denote the image of $W_{r}^{(n)}(\lambda, \mu)$ in the double coset $W_{\lambda} \backslash W_{r} / W_{\mu}$. By Proposition 14, each correspondence

$$
w \mapsto V(\lambda, w \cdot \mu), \quad w \mapsto M(\lambda, w \cdot \mu)
$$

defines an injective map from the set $\overline{W_{r}^{(n)}(\lambda, \mu)}$ to the set of equivalence classes of finite-dimensional $Y\left(\mathfrak{s l}_{n}\right)$-modules.

Let $\leq$ denote the Bruhat ordering in $W_{r}$ and let $P_{w, x}(q)$ denote the KazhdanLusztig polynomial associated with the Weyl group $W_{r}$ ([KL]).

In the following theorem we state the multiplicity formula of $Y\left(\mathfrak{s l}_{n}\right)$. For simplicity, we only consider the essential cases when the roots of Drinfeld polynomials are integers.

Theorem 15. Let $\lambda, \mu \in P_{r, \mathbb{Z}}^{+}$and $w \in \overline{W_{r}^{(n)}(\lambda, \mu)}$.

(1) The family $\left\{V(\lambda, x \cdot \mu) \mid x \in \overline{W_{r}^{(n)}(\lambda, \mu)}, x_{L R} \geq w_{L R}\right\}$ is exactly the set of all simple $Y\left(\mathfrak{s l}_{n}\right)$-modules which appear as the composition factors of the standard tensor product module $M(\lambda, w \cdot \mu)$. Moreover, their multiplicities are expressed as

$$
[M(\lambda, w \cdot \mu), V(\lambda, x \cdot \mu)]=P_{w_{L R}, x_{L R}}(1)
$$

for $x \in \overline{W_{r}^{(n)}(\lambda, \mu)}$.

(2) Conversely, the simple $Y\left(\mathfrak{s l}_{n}\right)$-module $V(\lambda, w \cdot \mu)$ is expressed as

$$
[V(\lambda, w \cdot \mu)]=\sum_{\substack{x \in W_{r}^{(n)}(\lambda, \mu) \\ x \geq w_{L R}}}(-1)^{\ell\left(w_{L R} w_{0}\right)-\ell\left(x w_{0}\right)} P_{x w_{0}, w_{L R} w_{0}}(1)[M(\lambda, x \cdot \mu)] .
$$

in the Grothendieck group of $\mathcal{C}_{Y\left(\mathfrak{s i}_{n}\right)}$, where $w_{0}$ denotes the longest element of $W_{r}$.

Proof. Due to the well-known Kazhdan-Lusztig conjecture ([BB, $\overline{B K}])$ and the translation principle ( $\mathrm{Jan}$ ), one has

$$
\begin{aligned}
& {[M(w \cdot \mu)]=\sum_{\substack{x \in W / W_{\mu} \\
x_{R} \geq w_{R}}} P_{w_{R}, x_{R}}(1)[L(x \cdot \mu)]} \\
& {[L(w \cdot \mu)]=\sum_{\substack{x \in W_{r} \\
x \geq w_{R}}}(-1)^{\ell\left(w_{R} w_{0}\right)-\ell\left(x w_{0}\right)} P_{x w_{0}, w_{R} w_{0}}(1)[M(x \cdot \mu)],}
\end{aligned}
$$

for $\mu \in P_{r}^{+}$and $w \in W / W_{\mu}$ in the Grothendieck group of the category $\mathcal{O}_{r}$ of $\mathfrak{g l}_{r}$, where $w_{R}$ denote the longest length representative in the coset $w W_{\mu}$. 
Then by (1.4.9), (1.4.10), Theorem 8 and Theorem 10, applying the exact functor $D_{\ell} \circ F_{\lambda}$, one has

$$
\begin{aligned}
& {[M(\lambda, w \cdot \mu)] }=\sum_{\substack{x \in W_{r}^{(n)}(\lambda, \mu) \\
x_{L R} \geq w_{R}}} P_{w_{R}, x_{L R}}(1)[V(\lambda, x \cdot \mu)] \\
& {[V(\lambda, w \cdot \mu)] }=\sum_{\substack{x \in W_{r}^{(n)}(\lambda, \mu) \\
x \geq w_{L R}}}(-1)^{\ell\left(w_{L R} w_{0}\right)-\ell\left(x w_{0}\right)} P_{x w_{0}, w_{L R} w_{0}}(1)[M(\lambda, x \cdot \mu)] \\
&
\end{aligned}
$$

in the Grothendieck group of $\mathcal{C}_{Y\left(\mathfrak{s t}_{n}\right)}$. Hence (2) is proved and (1) follows form Proposition 14 and (3.2.5).

Remark 16. If $x_{R} \geq w\left(\right.$ resp. $\left.x_{L} \geq w\right)$, then $x_{R} \geq w_{R}$ and $P_{w, x_{R}}(q)=P_{w_{R}, x_{R}}(q)$ (resp. $x_{L} \geq w_{L}$ and $P_{w, x_{L}}(q)=P_{w_{L}, x_{L}}(q)$ ), where $w_{L}$ denotes the longest length representative in the coset $W_{\lambda} w$ (see [Hum, Corollary 7.14], for example). Hence it follows that $P_{w, x_{L R}}(q)=P_{w_{R}, x_{L R}}(q)=P_{w_{L R}, x_{L R}}(q)$.

Remark 17. Let $\lambda-\rho$ and $\mu-\rho$ are both dominant integral weights, one can obtain the resolution of $V(\lambda, \mu)$ by applying the exact functor $D_{\ell} \circ F_{\lambda}$. This provides an alternative proof of the resolutions of the simple elementary module (NT2]) constructed by Cherednik (Che $)$. A generalization of such resolutions is possible to some extent by using the generalized BGG resolution obtained in [G.] (see [S] for the corresponding statement in the case of the degenerate affine Hecke algbera).

\section{Proof of Proposition 7}

Let $M$ be an $\mathcal{H}_{\ell}$-module and $U \subset M$ be a simple $W_{\ell}$-module such that $D_{\ell}(U) \neq 0$ as in the proposition. The proof is divided into 4 parts.

4.1. Let $\tilde{\mathcal{C}}_{\mathcal{H}_{\ell}}$ be the category of $\mathcal{H}_{\ell}$-modules who decompose into (possibly infinite) direct sum of finite-dimensional $W_{\ell}$-modules. Let $\tilde{\mathcal{C}}_{Y\left(\mathfrak{g l}_{n}\right)}$ be the category of $Y\left(\mathfrak{g l}_{n}\right)$ modules who decompose into (possibly infinite) direct sum of finite-dimensional $\mathfrak{g l}_{n}$-modules. Extend the Drinfeld functor $D_{\ell}$ to the functor from the category $\tilde{\mathcal{C}}_{\mathcal{H}_{\ell}}$ to the category $\tilde{\mathcal{C}}_{Y\left(\mathfrak{g l}_{n}\right)}$. It is easy to see that the extended functor $D_{\ell}$ is still an exact functor.

Let $\nu=\sum_{i=1}^{n} \nu_{i} \epsilon_{i}$ be the partition such that $U \cong U\left(\nu^{\prime}\right)$, where $\nu^{\prime}$ denotes the transpose of $\nu$ as before. Define an $W_{\ell}$-module

$$
J_{W}(\nu):=\mathbb{C}\left[W_{\ell}\right] \otimes_{\left(\mathbb{C}\left[W_{\nu_{1}}\right] \otimes \ldots \otimes \mathbb{C}\left[W_{\nu_{n}}\right]\right)}\left(\mathbb{C} 1_{\text {sign }, \nu_{1}} \otimes \ldots \otimes \mathbb{C} 1_{\text {sign }, \nu_{n}}\right),
$$

where $\mathbb{C} \mathbf{1}_{\text {sign, } \nu_{i}}$ is one-dimensional representation of $\mathbb{C}\left[W_{\nu_{i}}\right]$ such that $s_{i} \cdot \mathbf{1}_{\text {sign }, \nu_{i}}=$ $-\mathbf{1}_{\text {sign }, \nu_{i}}$. It is well-known that there exists a surjective homomorphism $\varphi_{W}$ : $J_{W}(\nu) \rightarrow U\left(\nu^{\prime}\right)$ of $W_{\ell}$-modules. Let $J(\nu)$ be an $\mathcal{H}_{\ell}$-module defined by

$$
J(\nu):=\mathcal{H}_{\ell} \otimes_{\mathbb{C}\left[W_{\ell}\right]} J_{W}(\nu) .
$$

Then $J(\nu)$ is an object of $\tilde{\mathcal{C}}_{\mathcal{H}_{\ell}}$. Let $\varphi: J(\nu) \rightarrow M$ be the surjective $\mathcal{H}_{\ell^{-}}$-homomorphism induced by $\varphi_{W}$. Then, the $Y\left(\mathfrak{g l}_{n}\right)$-homomorphism

$$
D_{\ell}(\varphi): D_{\ell}(J(\nu)) \longrightarrow D_{\ell}(M)
$$

is surjective and $D_{\ell}(\varphi)\left(J_{W}(\nu)\right)=D_{\ell}(U)$. Hence it is suffice to prove that

$$
D_{\ell}(J(\nu))=Y\left(\mathfrak{g l}_{n}\right) \cdot D_{\ell}\left(J_{W}(\nu)\right) .
$$


4.2. Recall the filterations on $\mathcal{H}_{\ell}$ and $Y\left(\mathfrak{g l}_{n}\right)$. For a gr $\mathcal{H}_{\ell}$-module $\bar{M}$, let $\bar{D}_{\ell}(\bar{M})$ denote the $\operatorname{gr} Y\left(\mathfrak{g l}_{n}\right)$-module $\bar{D}_{\ell}(\bar{M}):=\left(\bar{M} \otimes\left(\mathbb{C}^{n}\right)^{\otimes \ell}\right) / \sum \operatorname{Im}\left(s_{i}+1\right)$, in which $U\left(\mathfrak{g l}_{n}[t]\right) \cong \operatorname{gr} Y\left(\mathfrak{g l}_{n}\right)$ acts as

$$
E_{i j} \otimes t^{r} \mapsto \sum_{a=1}^{\ell} \bar{y}_{a}^{r} \otimes \tau_{a}\left(E_{i j}\right) .
$$

Then $\bar{D}_{\ell}$ defines an exact functor from the category of gr $\mathcal{H}_{\ell}$-modules who decomposes into direct sum of finite-dimensional $W_{\ell}$-modules to the category of $U\left(\mathfrak{g l}_{n}[t]\right)$ modules decomposes into direct sum of finite-dimensional $\mathfrak{g l}_{n}$-modules.

Now introduce a filteration on $J(\nu)$ by the followings;

$$
F_{-1} J(\nu)=0, F_{0} J(\nu)=J_{W}(\nu), F_{i} J(\nu)=\left(F_{i} \mathcal{H}_{\ell}\right) \cdot\left(F_{0} J(\nu)\right) \quad(i \in \mathbb{N}) .
$$

Let $\bar{J}(\nu)$ denote the the corresponding graded module of $J(\nu)$. Then, as a gr $\mathcal{H}_{\ell^{-}}$ module,

$$
\begin{aligned}
\bar{J}(\nu) & =\operatorname{gr} \mathcal{H}_{\ell} \cdot J_{W}(\nu) \\
& \cong\left(\mathbb{C}\left[\bar{y}_{1}, \ldots, \bar{y}_{\ell}\right] \otimes \mathbb{C}\left[W_{\ell}\right]\right) \otimes_{\mathbb{C}\left[W_{\ell}\right]} J_{W}(\nu) .
\end{aligned}
$$

In particular, $\bar{J}(\nu) \cong \mathbb{C}\left[\bar{y}_{1}, \ldots, \bar{y}_{\ell}\right] \otimes_{\mathbb{C}} J_{W}(\nu)$ as a $\mathbb{C}$-vector space.

Introduce a filteration on $D_{\ell}(J(\nu))$ induced from that of $J(\nu)$;

$$
F_{i} D_{\ell}(J(\nu)):=D_{\ell}\left(F_{i} J(\nu)\right) \text {. }
$$

By Proposition 5, one can easily check that $F_{i} Y\left(\mathfrak{g l}_{n}\right) \cdot F_{j} D_{\ell}(J(\nu)) \subset F_{i+j} D_{\ell}(J(\nu))$. Let $\operatorname{gr} D_{\ell}(\bar{J}(\nu))$ denote the corresponding graded module of $D_{\ell}(J(\nu))$. Then, by Proposition 5 and the fact that $\bar{D}_{\ell}$ is exact, one has

$$
\operatorname{gr} D_{\ell}(J(\nu)) \cong \bar{D}_{\ell}(\bar{J}(\nu))
$$

as a $\operatorname{gr} Y\left(\mathfrak{g l}_{n}\right) \cong U\left(\mathfrak{g l}_{n}[t]\right)$-module. Now 4.1.1) is reduced to the following proposition.

Proposition 18. As a $U\left(\mathfrak{g l}_{n}[t]\right)$-module,

$$
\bar{D}_{\ell}(\bar{J}(\nu))=U\left(\mathfrak{g l}_{n}[t]\right) \cdot \bar{D}_{\ell}\left(J_{W}(\nu)\right),
$$

where $\bar{D}_{\ell}\left(J_{W}(\nu)\right)$ denotes the image of $J_{W}(\nu) \subset \bar{J}(\nu)$ in $\bar{D}_{\ell}(\bar{J}(\nu))$.

4.3. As a preparation for the proof of Proposition 18, we shall first consider the simplest case when $\nu=\ell \epsilon_{i}$ for some $i$. Put $\bar{J}(\ell)=\bar{J}\left(\ell \epsilon_{i}\right)$. Then as a gr $\mathcal{H}_{\ell}$-module,

$$
\bar{J}(\ell) \cong \mathbb{C}\left[\bar{y}_{1}, \ldots, \bar{y}_{\ell}\right] \mathbf{1}_{\text {sign }},
$$

where $W_{\ell}$ act on the right-hand-side by $w \cdot\left(f \mathbf{1}_{\text {sign }}\right)=(-1)^{\ell(w)} w(f) \mathbf{1}_{\text {sign }}$.

Let $\mathbf{i}=\left(i_{1}, \ldots, i_{n}\right)$ be a permutation of $(1,2, \ldots, n)$. For $\gamma=\sum_{i=1}^{n} \gamma_{i} \epsilon_{i} \in$ $\mathrm{Wt}\left(\left(\mathbb{C}^{n}\right)^{\otimes \ell}\right)$, let

$$
u_{\mathbf{i}}(\gamma):=u_{i_{1}}^{\otimes \gamma_{i_{1}}} \otimes u_{i_{2}}^{\otimes \gamma_{i_{2}}} \otimes \ldots \otimes u_{i_{n}}^{\otimes \gamma_{i_{n}}} \in\left(\mathbb{C}^{n}\right)^{\otimes \ell} .
$$

Lemma 19. For a fixed permutation $\mathbf{i}=\left(i_{1}, \ldots, i_{n}\right)$ of $(1,2, \ldots, n)$, the set

$$
\left\{\left[\bar{y}_{1}^{d_{1}} \bar{y}_{2}^{d_{2}} \ldots \bar{y}_{\ell}^{d_{\ell}} \mathbf{1}_{\text {sign }} \otimes u_{\mathbf{i}}(\gamma)\right] \mid \begin{array}{l}
\gamma=\sum_{i=1}^{n} \gamma_{i} \epsilon_{i} \in \mathrm{Wt}\left(\left(\mathbb{C}^{n}\right)^{\otimes \ell}\right) \\
d_{m} \geq d_{m+1} \text { if } \sum_{j=1}^{a-1} \gamma_{i_{j}}<m<\sum_{j=1}^{a} \gamma_{i_{j}} \\
\text { for some } a .
\end{array}\right\}
$$

forms a $\mathbb{C}$-basis of $\bar{D}_{\ell}(\bar{J}(\ell))$. 
Proof. Clearly

$$
\bar{D}_{\ell}(\bar{J}(\ell)) \cong \bigoplus_{\gamma \in \mathrm{Wt}\left(\left(\mathbb{C}^{n}\right) \otimes \ell\right)}\left(\bar{J}(\ell) \otimes\left[\left(\mathbb{C}^{n}\right)^{\otimes \ell}\right]_{\gamma}\right) / \sum \operatorname{Im}\left(s_{i}+1\right)
$$

as a $\mathbb{C}$-vector space. In the case when $\gamma=\ell \epsilon_{i}$ for some $i$, it is well-known that

$$
\left(\bar{J}(\ell) \otimes\left[\left(\mathbb{C}^{n}\right)^{\otimes \ell}\right]_{\ell \epsilon_{i}}\right) / \sum \operatorname{Im}\left(s_{i}+1\right)=\bigoplus_{d_{1} \geq \cdots \geq d_{\ell}} \mathbb{C}\left[\bar{y}_{1}^{d_{1}} \ldots \bar{y}_{\ell}^{d_{\ell}} \mathbf{1}_{\text {sign }} \otimes u_{i}^{\otimes \ell}\right] .
$$

For a general weight $\gamma \in \mathrm{Wt}\left(\left(\mathbb{C}^{n}\right)^{\otimes \ell}\right)$, notice that the correspondence $w \mapsto w \cdot u_{\mathbf{i}}(\gamma)$ defines an isomorphism $\mathbb{C}\left[W_{\ell} / W_{\gamma_{i_{1}}} \times \cdots \times W_{\gamma_{i_{n}}}\right] \cong\left[\left(\mathbb{C}^{n}\right)^{\otimes \ell}\right]_{\gamma}$. On the other hand, $\bar{J}(\ell) \cong \bar{J}\left(\gamma_{i_{1}}\right) \otimes \ldots \otimes \bar{J}\left(\gamma_{i_{n}}\right)$ as a $W_{\gamma_{i_{1}}} \times \cdots \times W_{\gamma_{i_{n}}}$-module. Hence, by using the same argument as in the Proposition 1 , the statement reduces to (4.3.2).

For $k \in\{1, \ldots, n\}$, let $U\left(\mathfrak{g l}_{n}[t]\right)_{k}$ denote the subalgebra of $U\left(\mathfrak{g l}_{n}[t]\right)$ generated by the elements $E_{i k} \otimes t^{r}\left(i=1, \ldots, n, r \in \mathbb{Z}_{\geq 0}\right)$.

Lemma 20. For any $k \in\{1, \ldots, n\}$,

$$
\bar{D}_{\ell}(\bar{J}(\ell))=U\left(\mathfrak{g l}_{n}[t]\right)_{k} \cdot\left[\mathbf{1}_{\text {sign }} \otimes u_{k}^{\otimes \ell}\right] .
$$

Proof. Let $\mathbf{i}=\left(i_{1}, \ldots, i_{n}\right)=(1,2, \ldots, k-1, k+1, k+2 \ldots, n, k)$. For $m=$ $0,1, \ldots, \ell$, let $\bar{D}_{\ell}(\bar{J}(\ell))_{k, m}$ be the subspace of $\bar{D}_{\ell}(\bar{J}(\ell))$ spanned by the vectors of the form (4.3.1) such that $d_{j}=0$ for all $j>m$. We prove by inducition on $m$ that $\bar{D}_{\ell}(\bar{J}(\ell))_{k, m} \subset U\left(\mathfrak{g l}_{n}[t]\right)_{k} \cdot\left[\mathbf{1}_{\text {sign }} \otimes u_{k}^{\otimes \ell}\right]$.

Let $m=0$. Then for any $\gamma=\sum_{i=1}^{n} \gamma_{i} \epsilon_{i} \in \mathrm{Wt}\left(\left(\mathbb{C}^{n}\right)^{\otimes \ell}\right)$,

$$
\begin{aligned}
& \left(E_{i_{1} k}\right)^{\gamma_{i_{1}}}\left(E_{i_{2} k}\right)^{\gamma_{i_{2}}} \ldots\left(E_{i_{n-1} k}\right)^{\gamma_{i_{n-1}}} \cdot\left[\mathbf{1}_{\text {sign }} \otimes u_{k}^{\otimes \ell}\right] \\
& \equiv \frac{\ell !}{\gamma_{k} !}\left[\mathbf{1}_{\text {sign }} \otimes u_{\mathbf{i}}(\gamma)\right],
\end{aligned}
$$

where $E_{i j}=E_{i j} \otimes 1 \in \mathfrak{g l}_{n}[t]$. Hence $\bar{D}_{\ell}(\bar{J}(\ell))_{k, 0} \subset U\left(\mathfrak{g l}_{n}[t]\right)_{k} \cdot\left[\mathbf{1}_{\text {sign }} \otimes u_{k}^{\otimes \ell}\right]$.

Next let $m>0$ and suppose that $\bar{D}_{\ell}(\bar{J}(\ell))_{k, m-1} \subset U\left(\mathfrak{g l}_{n}[t]\right)_{k} \cdot\left[\mathbf{1}_{\text {sign }} \otimes u_{k}^{\otimes \ell}\right]$. Let $a$ be the integer such that $\sum_{j=1}^{a-1} \gamma_{i_{j}}<m \leq \sum_{j=1}^{a} \gamma_{i_{j}}$. Then, one has

$$
\begin{aligned}
& \left(E_{i_{a} k} \otimes t^{d_{m}}\right) \cdot\left[\left(\bar{y}_{1}^{d_{1}} \bar{y}_{2}^{d_{2}} \ldots \bar{y}_{m-1}^{d_{m-1}} \mathbf{1}_{\text {sign }}\right) \otimes u_{\mathbf{i}}\left(\gamma-\epsilon_{i_{a}}+\epsilon_{k}\right)\right] \\
& \equiv(\ell-m+1)\left[\left(\bar{y}_{1}^{d_{1}} \bar{y}_{2}^{d_{2}} \ldots \bar{y}_{m}^{d_{m}} \mathbf{1}_{\text {sign }}\right) \otimes u_{\mathbf{i}}(\gamma)\right]
\end{aligned}
$$

for $d_{m}>0$. Here the equality holds modulo $\bar{D}_{\ell}(\bar{J}(\ell))_{k, m-1}$ if $a=n$ (i.e, $i_{a}=k$ ). Hence by inducition hypothesis, $\bar{D}_{\ell}(\bar{J}(\ell))_{k, m} \subset U\left(\mathfrak{g l}_{n}[t]\right)_{k} \cdot\left[\mathbf{1}_{\text {sign }} \otimes u_{k}^{\otimes \ell}\right]$.

4.4. Let turn ourselves back to the proof of Proposition 18. The following lemma is an analogue of Proposition 1 .

Lemma 21. As a $\mathfrak{g l}_{n}[t]$-module,

$$
\bar{D}_{\ell}(\bar{J}(\nu)) \cong \bar{D}_{\nu_{1}}\left(\bar{J}\left(\nu_{1}\right)\right) \otimes \bar{D}_{\nu_{2}}\left(\bar{J}\left(\nu_{2}\right)\right) \otimes \ldots \otimes \bar{D}_{\nu_{n}}\left(\bar{J}\left(\nu_{n}\right)\right) .
$$

Now let us complete the proof of Proposition 18. Let $v_{i}=\left[\mathbf{1}_{\text {sign }} \otimes u_{i}^{\otimes \nu_{i}}\right]$ and $v_{[k]}:=v_{k} \otimes \ldots \otimes v_{n}$. Notice that $U\left(\mathfrak{g l}_{n}[t]\right)_{k} \cdot v_{a} \subset \mathbb{C} v_{a}$ if $a \neq k$. Hence one can show by inducition on $k$ that

$$
U\left(\mathfrak{g l}_{n}[t]\right)_{1} \cdot v_{1} \otimes U\left(\mathfrak{g l}_{n}[t]\right)_{2} \cdot v_{2} \otimes \ldots \otimes U\left(\mathfrak{g l}_{n}[t]\right)_{k} \cdot v_{k} \otimes \mathbb{C} v_{[k+1]} \subset U\left(\mathfrak{g l}_{n}[t]\right) \cdot v_{[1]} .
$$

Now Proposition 18 follows from Lemma 20. 
Acknowledgement I am deeply gratefull to Akihiro Tsuchiya for his constant support, including valuable advice and carefully reading of the manuscript. I am also gratefull to Tomoki Nakanishi for his encouragement and valuable advice. I whould like to thank Takeshi Suzuki for the collaboration in [AS], which lead to this work.

\section{REFERENCES}

[AK] T. Akasaka and M. Kashiwara. Finite-dimensional representations of quantum affine algebras. Publ. Res. Inst. Math. Sci., 33(5):839-867, 1997. (math.QA/9703028).

[AS] T. Arakawa and T. Suzuki. Duality between $\mathfrak{s} l_{n}(\mathbb{C})$ and the degenerate affine Hecke algebra. to appear in J. Alg. (math.QA/971037).

[BB] A. Beilinson and J. Bernstein. Localisation de g-modules. C. R. Acad. Sc. Paris, 21:152-154, 1987.

[BGHP] D. Bernard, M. Gaudin, F. D. M. Haldane, and V. Pasquier. Yang-Baxter equation in long-range interacting systems. J. Phys. A: Math. Gen., 26(5219-5236), 1993.

[BK] J. L. Brylinski and M. Kashiwara. Kazhdan-Lusztig conjecture and holonomic systems. Invent. Math., 64(3):387-410, 1981.

[CG] N. Chriss and V. Ginzburg. Repesentation theory and complex geometry. Birkhäuser, 1997.

[Che] I. V. Cherednik. A new interpretations of Gelfand-Tzetlin basis. Duke. Math., 54:563$578,1987$.

[CP1] V. Chari and A. Pressley. Quantum affine algebras. Comm. Math. Phys., 142(2):261285, 1991.

[CP2] V. Chari and A. Pressley. Quantum affine algebras and affine Hecke algebras. Pacific J. Math., 174(2):295-326, 1996.

[CP3] V. Chari and A. Pressley. Yangians: their representations and characters. Acta Appl. Math., 44(1-2):39-58, 1996.

[D1] V. G. Drinfeld. Hopf algebras and the quantum Yang-Baxter equation. Soviet Math. Dokl., 32:254-258, 1985.

[D2] V. G. Drinfeld. Degenerate affine Hecke algebras and Yangians. Funct. Anal. Appl., 20(1):62-64, 1986.

[D3] V. G. Drinfeld. A new realization of Yangians and of quantized affine algebras. Soviet Math. Dokl., 36:212-216, 1988.

[FH] W. Fulton and J. Harris. Repesentation theory. Springer-Verlag, 1991.

[Gin] V. A. Ginzburg. Proof of the Deligne-Langlands conjecture. Soviet. Math. Dokl., 35(2):304-308, 1987.

[GJ] O. Gabber and A. Joseph. On the Bernstein-Gelfand-Gelfand resolution and the Duflo sum formula. Compos. Math., 43:261-302, 1981.

[Hum] J. E. Humphreys. Reflection groups and Coxter groups. Number 29 in Cambridge studies in advanced mathematics. Cambridge, 1990.

[Jan] J. C. Jantzen. Moduln mit einerm höchsten Gewicht. Number 750 in Lecture Notes in Mathematics. Springer-Verlag, 1983.

[KL] D. Kazhdan and G. Lusztig. Representations of Coxter groups and Hecke algebras. Invent. Math., 2(53):165-184, 1979.

[Lus] G. Lusztig. Affine Hecke algebras and their graded version. J. Amer. Math. Soc., 2(3):599-635, 1989.

[MNO] A. Molev, M. Nazarov, and G. Olshanski. Yangians and classical Lie algebras. Russian Math. Surveys, 51(2):205-283, 1996.

[Mol] A. Molev. Finite-dimensional irreducible representations of twisted Yangians. prerpint, math.QA/9711022.

[NT2] M. Nazarov and V. Tarasov. On irreduciblity of tensor products of Yangian modules. Internat. Math. Res. Notices, (3):125-150, 1998. (math.QA/9712004).

[NT1] M. Nazarov and V. Tarasov. Representations of Yangians with Gelfand-Zetlin bases. J. Reine Angew. Math., 496:181-212, 1998. (math.QA/9502008). 
[Ro] J. D. Rogawski. On modules over the Hecke algebra of a p-adic group. Invent. Math., 79:443-465, 1985.

[S] T. Suzuki. Rogawski's conjecture of the Jantzen filtlation for the degenerate affine Hecke algebra of type A. preprint, math.QA/9804012.

[Zel] A. Z. Zelevinsky. Induced representations of reductive p-adic groups II. Ann. Sci. Ecole Norm. Sup., $4^{e}$, Serie 13:165-210, 1980.

Graduate school of Mathematics, Nagoya University, Chikusa-Ku, Nagoya, 464-8602, JAPAN

E-mail address: tarakawa@math.nagoya-u.ac.jp 